\title{
Psoriasis Vulgaris Successfully Treated with Goeckerman Treatment at Home: A Patient and Physician's Experience
}

\author{
Vidhatha Reddy · Shawn Thomas - Quinn Thibodeaux • \\ Bridget Myers · Tina Bhutani · Wilson Liao
}

Received: February 10, 2020 / Published online: March 19, 2020

(C) The Author(s) 2020

\begin{abstract}
Goeckerman therapy is a highly effective treatment regimen for moderate-to-severe psoriasis. It involves regular exposure to ultraviolet $\mathrm{B}$ radiation and the application of crude coal tar. To our knowledge, only three centers in the USA currently offer a formal Goeckerman therapy treatment program; thus, access to this therapy is geographically limited. In this article, a motivated patient discusses his experience with generalized plaque psoriasis. This patient, while living in a Goeckerman-inaccessible area, deferred treatment with biologics and outpatient phototherapy to develop a modified Goeckerman regimen for at-home use. This home regimen, which did not involve the use of prescription-strength medications, resulted in full clearance of his psoriasis. We also discuss the patient's case from the perspective of a dermatology treatment team that has reviewed his experience.
\end{abstract}

The article is part of the Topical Collection on Patient.

Enhanced Digital Features To view enhanced digital features for this article go to https://doi.org/10.6084/ m9.figshare.11898216.

V. Reddy - Q. Thibodeaux · B. Myers - T. Bhutani . W. Liao ( $\square)$

Department of Dermatology, University of

California, San Francisco, San Francisco, CA, USA

e-mail: wilson.liao@ucsf.edu

S. Thomas

Citizen Scientist, Chicago, IL, USA
Keywords: Coal tar; Goeckerman therapy; Heliotherapy psoriasis; Home therapy; Phototherapy

\section{Key Summary Points}

Gockerman therapy is a well-established and efficacious treatment for plaque psoriasis that involves the use of topical crude coal tar (CCT) and phototherapy with ultraviolet B light.

Many patients are unable to receive officebased Gockerman therapy due to geographic limitations.

This patient-provider perspective article demonstrates how one patient was able to use over-the-counter CCT products and heliotherapy to treat his plaque psoriasis with a home-based, modified Goeckerman regimen under the supervision of his dermatologist.

Home-based therapies for plaque psoriasis that are modified from evidence-based techniques may be safely adapted for patients who are otherwise unable to receive treatment.

Collaboration between physicians and patients is crucial for developing new, creative treatment regimens. 


\section{PATIENT'S EXPERIENCE}

My psoriasis first introduced itself as a peculiar, dry patch on my left shin. It seemed innocuous at first. I dismissed it, hoping the dry skin would resolve on its own. But after a couple weeks, it started to itch almost every night, flaking off a thin layer of dead skin when scratched. This was odd and disconcerting. I decided to take more decisive action. I doused it with layers of various topical creams: lotion, cocoa butter, and petroleum jelly. But the dry patch endured.

And then-to my horror-multiplied.

Over 7 months, from October 2017 to May 2018 , reddish coin-shaped patches peppered my shin and calves on my left leg, then on my right leg. The patches then crawled up, popping up on my inner thighs, chest, and elbows. I had hoped the warmer months would help alleviate my condition. But on the first week of May, yet another red patch appeared. This time on my nose, like a big teenage pimple on my 34-yearold face.

I made an appointment with a local dermatologist. He took a punch biopsy from my left leg, and the results led to the conclusion that I had plaque psoriasis, an incurable autoimmune disease. Psoriasis was not directly life-threatening, as far as incurable diseases go. I tried to remind myself that I got the luckier end of the deal, but "incurable" is such a hope-snuffing word. A slight melancholy usually descended after a shower-when my red plaques were at their brightest. I attentively examined the plaque on my face, looking for any sign of improvement. Any fleeting thought of changing my circumstances was immediately reined in by the weight of the word "incurable."

Living with psoriasis is an exercise in expert cover-ups. Pants and long sleeve shirts-even in the summer-were my staple outfit, and my uniform for recreational flag football tournaments now included knee-high socks. The raised, reddish plaques itched intensely, often at night. And every couple of days, a thin layer of skin would rapidly build up on my plaques and then slough off and crumble, leaving trails of skin flakes behind on my clothes and bedsheets.
At my next appointment, the dermatologist prescribed clobetasol $0.05 \%$, a potent topical corticosteroid cream. I applied it on my plaques twice daily for 2 weeks, but my plaques remained fairly unchanged. I understood that should the corticosteroids fail, the dermatologist would next consider a biologic treatment, such as Humira ${ }^{\circledR}$ (adalimumab) or Taltz (ixekizumab). I was generally wary of this option for a number of reasons: life-long dependence, potential immunosuppressant side effects, and an expensive price tag. For me, this seemed like a last resort option; I wanted to first exhaust other reasonable alternatives.

I was fairly open to telling my friends and family about my diagnosis and, in doing so, received two unexpected benefits. First, they shared with me their experiences with psoriasis-either those of their own or those of a family member who had the disease. Before I received the diagnosis, I did not think I personally knew anyone who had psoriasis; I was surprised to learn how far from alone I actually was. Second, they shared stories of different remedies that they had tried, including what seemed to help and what didn't. For 8 months, my psoriasis showed no signs of abatement. Their stories restored some hope that perhaps a treatment could at least reduce the number of my plaques.

Through personal conversations and reading online articles, I compiled a list of potential psoriasis treatments: a vegan diet, gluten-free diet, keto diet, a vegan and keto diet, alkaline water, potent ingestible probiotics, cold showers, a probiotic skin spray, all sorts of different moisturizers, and an Ayurvedic solution from India. I downloaded an app called Imagine that helped to organize time-lapsed photos of different parts of the body. Through the app, I could take daily pictures of one body part, such as my left leg, and then easily swipe through the time-stamped photos to determine whether there were any noticeable changes in the appearance of the plaques. Using my body as a live science experiment, I introduced and tested each potential psoriasis treatment and documented the results. After force-drinking daily cups of concentrated probiotic solutions, temporarily dying my skin red from the Ayurvedic 
solution, nearly starving myself for 2 weeks on a keto-vegan diet that consisted mostly of pumpkin seeds, and gathering my nerves before every ice-cold shower, my psoriasis plaquesshowing complete disdain for my efforts-remained unchanged. The cycle continued without disruption: flare up, skin buildup, itch all over, shedding of a skin layer, and repeat. Disheartened, I had to admit that my "throw the kitchen sink" strategy had failed.

I was disappointed yet still felt duty-bound to continue. I took a sober, realistic attitude toward my psoriasis, under no illusion that the chances of finding a non-prescriptive remedy to reduce the severity of my disease was anywhere within the realm of possibility. But I was fueled by the simple thrill of the challenge and the obligation to try.

I decided to take a more thoughtful approach: to replace my Google searches with Google Scholar searches to delve down into the peer-reviewed medical literature and to better understand the pathology of the disease. When interesting online publications were blocked by pricey paywalls, I leaned on the kindness of physician and pharmacist friends at academic hospitals and asked whether they could send me the full-length articles. I read through study after study, cataloging the conclusions of various experiments as far back as the 1920s, drawing out the pathways of cytokine overexpression and forming hypotheses on the nature of the disease.

In late July 2018, I read an article published in 2013 by the University of California, San Francisco (UCSF) that had caught my eye, entitled The Goeckerman Regimen for the Treatment of Moderate to Severe Psoriasis. UCSF has one of three clinics in the USA that administers the Goeckerman regimen, and the reported statistics from this clinic were incredible. According to this article, "100\% of patients on Goeckerman therapy achieved PASI 75 [75\% improvement of Psoriasis Assessment of Severity Index (PASI) from baseline] by 3 months." I thought: $100 \%$ of patients responded to the treatment? After all my experimentation, I had not witnessed a glimmer of a response. And here, at this clinic, every patient did. The article continued: “...patients on the most potent biologics achieved only $67-68 \%$ PASI 75 in 3 months." The Goeckerman regimen was not only more effective than the leading biologic (as of 2013), but it even worked on patients who did not respond to biologic treatment. To top it off, the regimen had "an extremely safe toxicity profile with essentially no internal side effects." The article detailed a 24-step process on how to perform the Goeckerman regimen, which consisted of ten topical applications and phototherapy treatments. One Goeckerman session requires approximately $6 \mathrm{~h}$ to complete. The session is performed daily on the patient until the symptoms clear. The total treatment period can range anywhere between 3 and 8 weeks.

Despite the extraordinary promise of the therapy, there were several logistical and financial challenges: Should I take potentially 8 weeks off of work to pursue this? How expensive would it be to travel to San Francisco and find temporary housing? Would my health insurance cover the UCSF Goeckerman therapy? If not, how much would it cost out of pocket? As the questions piled up, a seditious thought arose. A thought that would render the current questions moot, but would open a new phase of my own research and experimentation: If this therapy is remarkably safe, how hard would it be to replicate it at home?

The bulk of Goeckerman studies were conducted in the 1970s and 1980s. From what I gathered, it might be possible to boil down the 24-step UCSF Goeckerman regimen to three essential elements: a topical coal tar application, plastic wrap occlusion, and exposure to narrowband ultraviolet B (UVB) light. Although coal tar and UVB light can independently help alleviate some psoriatic symptoms, there was a powerful synergistic therapeutic effect when used together. I set out to acquire each of the essential ingredients required to replicate a similar effect at home.

I bought a small jar of coal tar online and a roll of plastic wrap from my local grocery store. But the challenge was finding a narrowband UVB light source as home phototherapy units were exorbitantly expensive and often required a prescription. After brainstorming alternative options, I arrived at this-it turns out, incorrect-conclusion: If the Goeckerman therapy was 
invented in the 1920s, Dr. Goeckerman probably didn't use a phototherapy unit. He must have used the sun (Dr. Goeckerman had actually used medium pressure mercury arc lamps). I neglected to do a simple Google search to confirm my assumption and just-well-ran with it.

After an inquiry into UV radiation, I learned that the amount of UVB light from the sun that reaches the earth's surface is dependent on multiple factors, including geographical location (e.g., latitude), time of the year, time of the day, altitude, weather, air pollution, etc. At the time, I was traveling for work to the suburbs of Baltimore in late July. Based on my rough estimates, in order to harness the most heliotherapeutic radiation for Goeckerman, I needed to expose large swaths of my skin to the sun sometime between the hours of $11 \mathrm{a} . \mathrm{m}$. and 2 p.m. This inconveniently was smackdab in the middle of the workday. To properly test this improvised Goeckerman therapy, two words came to mind: "strategic lunchbreaks."

The coal tar had the color of brown mustard and a semi-solid consistency similar to petroleum jelly. At my hotel in Baltimore, I used latex gloves to rub a thin layer of the ointment onto my psoriasis plaques. I held one end of the plastic wrap in place with one hand, and then with the other I slowly unspooled the plastic wrap from the roll, circling and occluding most of my affected skin. Once the wrapping process was complete, I ripped the plastic wrap free from the roll and tucked the end under another plastic wrap swatch, securing it in place with a piece of tape. I slept overnight with the plastic occlusions wrapped around my legs and arms. I reasoned that this activity fulfilled the $4+\mathrm{h}$ plastic wrap occlusion wait time-a critical step in the UCSF Goeckerman regimen. The following morning, I unwrapped the plastic wrap and thoroughly washed off the coal tar from my skin with soap and water. The ointment had a faint smell of tar, but it was barely perceptible on my skin after the shower.

The next critical step was the UVB radiation exposure. I left work around noon for "lunch" and drove back to the hotel, which was luckily only a quick 5-min drive away. In my hotel room, I stripped down and changed into just a pair of shorts and flip flops, and with a couple towels draped over my shoulder, I headed back outside. As I passed the front desk, I heard a voice, "Sir, are you looking for the swimming pool?"

"No, no. Just the parking lot," I replied, giving a slight jerk of my head toward the main entrance.

"Oh...okay."

After an awkward pause, I gently nodded and walked through the sliding double doors.

A small field hugged the far end of the parking lot and a lone wooden picnic bench sat along the field's edge. I placed a large towel flat on top of the table and laid down on top of it, facing the sky. It was a clear day, and the unobstructed sun lit up my saddle-colored skin to a shade of chestnut, bespeckled with crimson red spots. I wore sunglasses to protect my eyes, but for additional protection, I folded a small hand towel and placed it over my face as well.

I knew that exposing large areas of bare unprotected skin to the noonday sun was against commonly held wisdom. There was even a warning label on my jar of coal tar that advised to "use caution in exposing skin to sunlight" after using the product. To mitigate the risk of sunburn, I used an equation that considered the daily UV index and my Fitzpatrick skin type to estimate the time it would take my skin to burn. To play it even safer, I arbitrarily multiplied that estimate by $10 \%$, which often came out to approximately $10 \mathrm{~min}$.

I lay out baking in the sun with my cellphone timer set for $10 \mathrm{~min}$. My eyes closed, I could feel a blanket of warm radiation gently press down on my body.

My timer went off. I reset it for another $10 \mathrm{~min}$ and flipped over, face down. After the timer went off a second time, I gathered my towels and headed back to the hotel. I rinsed off my skin in the shower, changed back into my button-down collared shirt and trousers, and drove back to work. That evening, I checked my skin. Luckily, there were no tell-tale signs of sunburn.

As often as the weather and my work schedule permitted, I repeated this procedure: apply coal tar and occlude skin in the evening; expose skin to UVB light at noon; and periodically evaluate for any sunburn or adverse 
reactions. After the first week, I noticed something remarkable. The psoriasis plaques had visibly flattened and turned a lighter shade of red with splotches of darkened skin. Something was happening-finally!

In mid-August, about 3 weeks into the improvised Goeckerman treatment, I made an appointment at my local dermatologist office. I wanted to get advice from a professional on whether my skin's unusual response to the treatment was an indication that the psoriasis plaques were healing. "No," the dermatology physician assistant said. "It hasn't healed. The way you can tell is if you close your eyes, run your fingers along the skin, and see whether you can feel a difference between the plaques and your normal skin."

"I see," I said, as I followed her instruction, "you can still feel some raised plaques, but it does look a lot different than it did just a few weeks ago."

I told her the story of my improvised Goeckerman treatment, but she remained skeptical. "I would recommend you try one of the biologics."

I squinted an eye into a slight grimace. "I want to see how this goes first before going down that route," I replied. "Can I ask thoughit seems you're skeptical of this type of phototherapy-why?"

"It's just I've seen patients do phototherapy at the office and it works for a while but then it often comes back. With a biologic, you don't have to see me again for another 6 months."

She gave me a prescription for another type of topical corticosteroid and said I could try to continue to do what I was doing if it seemed to be helping.

I never filled the prescription. After 3 more weeks on the home Goeckerman therapy, my psoriasis plaques were gone. There was no trace of my plaques on my arms and chest and thighs; it looked like normal skin. The plaques on my shin and calves were also gone, but had left behind darkened, hyperpigmented skin in their absence. The psoriasis plaque on my nose had healed as well. This was a fascinating detail: I did not want to experiment with my face so I never applied coal tar on my nose and covered up my face, nose included, with a towel when exposing my body to UVB light. Despite this, this plaque too disappeared.

To put it mildly, I was very satisfied with the results. But the words of the dermatology physician assistant stayed with me, "...it often comes back." For good measure, I continued the home Goeckerman therapy for another 4 weeks (Fig. 1).

Throughout the winter in Chicago, there was no trace of the plaques returning. When spring arrived, I took up the habit of a weekly 4-mile run at noon along the lake with my shirt offmy bare, exposed skin absorbing the heliotherapeutic UVB light. When the psoriasis plaques hadn't returned in about 1 year, I decided it was time to share my experience and further explore the feasibility of this therapy option for others.

I started by extending my gratitude to the physicians, researchers, and medical students who authored the publication on the UCSF Goeckerman regimen. The UCSF paper was instrumental in developing the home version of the therapy that ultimately healed my plaques. Dr. Wilson Liao, co-author of this article, replied to my thank you email and inquired for more details on my experience. We discussed the risks and promises of adapting the Goeckerman regimen for home use, and he encouraged me to share my story through a medical journal publication.

With only a sample size of one, I wanted more assurance that what worked for me could be safely and reliably repeated for other psoriasis patients. I shared a post on my Instagram with before and after pictures of my psoriasis, encouraging readers to contact me if they, or someone they knew, had psoriasis. Over dozens of calls and coffee chats, I heard stories of how my friends, former colleagues, and acquaintances had suffered from psoriasis, including the physical and emotion toll it caused over the years, how they thought through the available treatment options, and how they currently manage the disease. I shared my experience and the treatment I had used to send my psoriasis into full remission. If they were willing to try the home Goeckerman regimen, I asked if they could check in with me weekly so I could track their progress and results. 


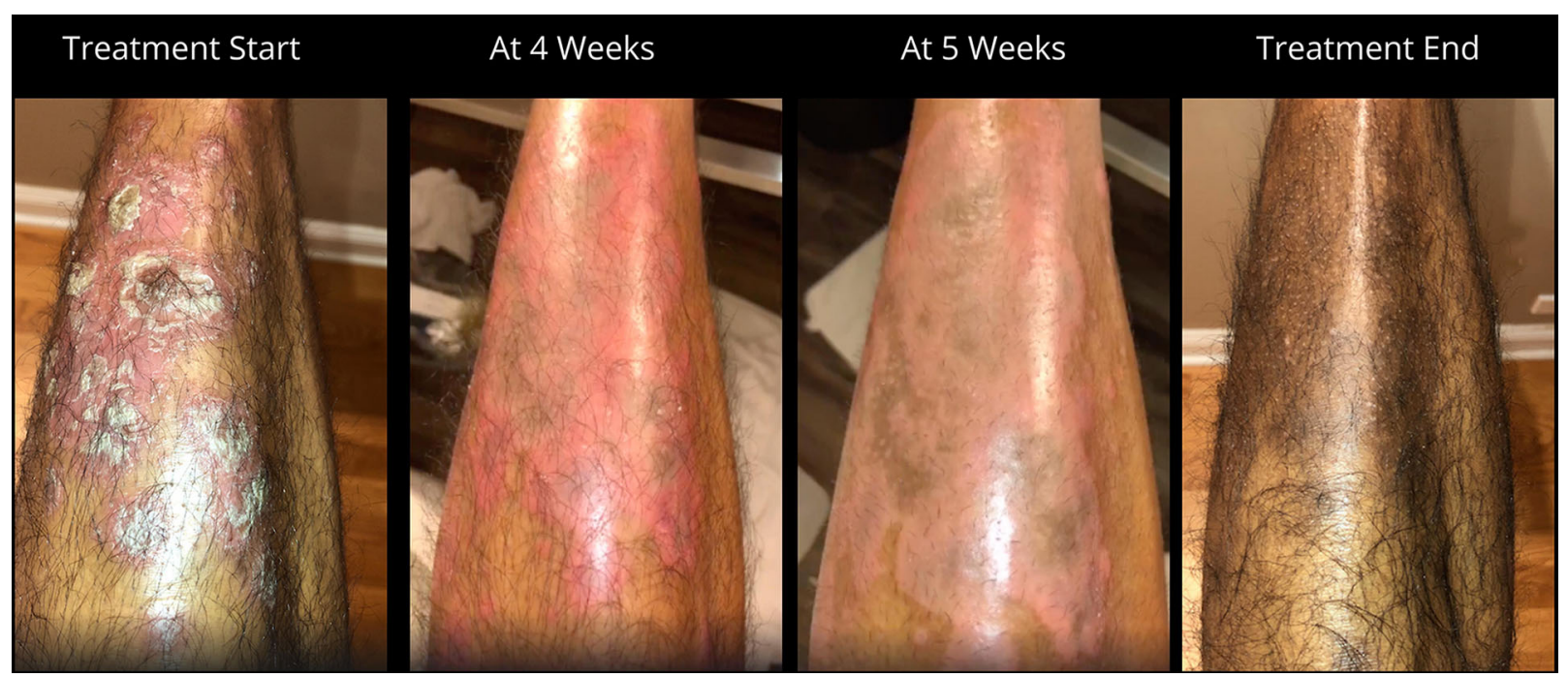

Fig. 1 Progression of plaque psoriasis treatment on left leg

Some who only had a mild form of psoriasis were less willing to put in the effort that the home Goeckerman treatment required. For the ones that attempted the treatment, the critical step to absorb UVB light was often obstructed by their work schedules or uncooperative weather. But for the ones that consistently adhered to the treatment, the results were impressive. One man in his mid-30 s had psoriasis for 4 years. He had been on Humira, a biologic, which helped to reduce most of his psoriasis plaques, but he still had plaques on his shins. He tried the home Goeckerman therapy-while continuing his Humira treatmentand after just 4 weeks, the psoriasis plaques on his shins showed what he estimated as $80 \%$ improvement.

\section{MEDICAL PROVIDER PERSPECTIVE}

Mr. Thomas first reached out to the clinical research team at the UCSF Psoriasis and Skin Treatment Center after performing his own personal research on the Goeckerman regimen. We thought it was fascinating that Mr. Thomas took the initiative to modify the Goeckerman regimen for home use, based on his personal situation and treatment preferences. For interested readers, we have provided a description of his protocol (Table 1), the over-the-counter materials used (Table 2), and the method of calculating UV light dosage from sun exposure (Table 3). Mr. Thomas has agreed to voluntarily share his experience on his own accord in order to potentially help other patients who may benefit from his approach.

Goeckerman therapy, originally developed in 1925 at the Mayo Clinic, has proven to be highly effective for the treatment of moderateto-severe psoriasis [1]. Although the mechanism of coal tar has not been definitively identified, some evidence suggests that polycyclic aryl hydrocarbons may be responsible for the majority of its therapeutic effect in psoriasis $[2,3]$. Goeckerman therapy has been used successfully in cases of treatment-resistant psoriasis and in patients who have contraindications to biologic medications [4, 5]. While useful for patients in these scenarios, Goeckerman therapy has traditionally been administered as an inpatient or formal outpatient treatment program, requiring patients to either live nearby or travel regularly to a treatment center. We were intrigued by Mr. Thomas' case as many patients who reach out to our center and are interested in pursuing Goeckerman therapy report an inability to do so because of geographic and scheduling limitations.

Despite these limitations, Goeckerman therapy remains one of the most effective treatments for plaque psoriasis. One study of UCSF 
Table 1 Our patient's approach to the Goeckerman regimen for home use

Evaluation and preparation

Application of coal tar

Heliotherapy
A. Confirmed with licensed dermatologist that he was an appropriate candidate for Goeckerman regimen. Patient qualified because he did not have a known history of adverse reactions to ambient sunlight or light therapy and was not taking any photosensitizing medications

B. Purchased the necessary materials (Table 2)

A. Applied generous amounts of coal tar gel to areas where psoriasis was present

B. Occluded coal tar gel with plastic wrap. (For hand psoriasis, gloves are typically used for occlusion. For scalp psoriasis, a shower cap is used. For foot psoriasis, a shower cap placed inside a sock is used)

C. Maintained occlusion for at least $4 \mathrm{~h}$. Performed application and occlusion prior to sleeping and wore occlusive materials overnight

D. After a minimum of $4 \mathrm{~h}$, removed occlusive materials and washed off tar in the shower with soap and water. Applied mineral oil on occasion to help remove coal tar

E. Used a variety of moisturizers as needed (Aquaphor ${ }^{\circledR}$, Vanicream ${ }^{\mathrm{TM}}$, and Cetaphil) following coal tar removal to prevent irritation and dryness

A. For heliotherapy, patient used the Environmental Protection Agency UVI to calculate the UVI based on zip code. He then used a formula (Table 3) based on Fitzpatrick skin type to calculate time-to-burn for his skin. Once time-to-burn was calculated, he arbitrarily began heliotherapy at $15 \%$ of time to burn. For example, for the first session, if the patient's calculated time-to-burn was $100 \mathrm{~min}$, he would spend $15 \mathrm{~min}$ exposed to the sun while lying supine and 15 min exposed while lying prone. The patient wore shorts and covered his face, but exposed all other areas of his body given that psoriasis was present in multiple locations

B. Monitored for over-exposure to UVB radiation. Although the patient never experienced significant erythema, irritation, or burning, he planned to either reduce or stop exposure to UV radiation if these side effects occurred. Patient also followed-up with dermatologist following initiation of home Goeckerman therapy once during treatment and once after treatment following symptom resolution

$U V B$ Ultraviolet $\mathrm{B}, U V I$ ultraviolet index

Table 2 Modified home Goeckerman therapy materials

\begin{tabular}{ll}
\hline Purpose & Material(s) \\
\hline Crude coal tar (CCT) & MG217 psoriasis 2\% coal tar multi-symptom ointment \\
Occlusion & MG217 psoriasis medicated conditioning 3\% coal tar formula shampoo \\
& Plastic wrap \\
& Shower cap (scalp psoriasis) \\
& Plastic gloves (hand psoriasis) \\
& Disposable socks/booties (foot psoriasis) \\
\hline
\end{tabular}


Table 3 Heliotherapy calculation used by patient

\begin{tabular}{llll}
\hline Skin type & Unexposed skin color & Reaction to sun exposure & Time to burn $(\mathbf{m i n})$ \\
\hline I & White & Always burns, never tans & $(200 \times 2.5) /(3 \times$ UVI $)$ \\
II & White & Always burns, minimal tan & $(200 \times 3) /(3 \times$ UVI $)$ \\
III & White to olive & Burns minimally, gradually tans & $(200 \times 4) /(3 \times$ UVI $)$ \\
IV & Light brown & Burns minimally, tans well & $(200 \times 5) /(3 \times \mathrm{UVI})$ \\
V & Brown & Very rarely burns, tans profusely & $(200 \times 8) /(3 \times \mathrm{UVI})$ \\
VI & Dark brown to black & Never burns, tans deeply & $(200 \times 15) /(3 \times \mathrm{UVI})$ \\
\hline
\end{tabular}

Goeckerman patients found that $100 \%$ of patients achieved 75\% improvement in the Psoriasis Area and Severity Index score (PASI 75) following treatment [6]. Another study examining 300 patients in both Baylor University and UCSF's Goeckerman centers found that $100 \%$ of psoriasis patients achieved 90\% improvement in the PASI score (PASI 90), with $73 \%$ of patients remaining in remission for at least 12 months [7]. Goeckerman therapy, however, has steadily lost favor due to the time commitment required from patients, typically 4-6 h/day for 6 weeks in a clinical setting. This is one of the main reasons why only a handful of clinics in the USA currently offer Goeckerman therapy. Mr. Thomas' case demonstrates that an excellent skin response is possible when utilizing a modified at-home Goeckerman regimen. This regimen also provides an opportunity to significantly increase the availability of Goeckerman therapy to patients who are unable to pursue formal therapy due to geographic, financial, or time constraints. Additionally, office-based Goeckerman therapy has also been used in other dermatologic conditions apart from psoriasis, including atopic dermatitis and prurigo nodularis $[8,9]$. The modified home Goeckerman therapy presented here may also prove to be successful in treating these other dermatologic conditions.

There are, however, potential risks to this approach that should be discussed. In formal Goeckerman therapy programs, patients are examined daily by a dermatologist to evaluate treatment progression and monitor for burns secondary to phototherapy. This allows for subsequent titration of phototherapy dosage if necessary. An at-home regimen inherently involves less monitoring and may result in unintentional injury, although Mr. Thomas did not experience any adverse events. Dermatologists should educate patients on the photosensitizing potential of crude coal tar (CCT) and on how to monitor for signs of burning and stinging, particularly for patients with Fitzpatrick skin types I-IV. Furthermore, it is also important to educate patients on normal skin changes throughout this process, including the post-inflammatory hyperpigmentation seen in $\mathrm{Mr}$. Thomas' case and the potential need for maintenance therapy to prevent remission. It is also crucial to maintain open communication throughout the process if patients wish to initiate heliotherapy while using concomitant CCT. Adverse reactions to ultraviolet A in sunlight may not manifest until $48 \mathrm{~h}$ following exposure [10]. One option to minimize this risk would be to utilize a home phototherapy unit prescribed by a dermatologist. Another potential concern relates to the use of topical corticosteroids in conjunction with CCT, which is often done at formal Goeckerman therapy centers under the supervision of a clinician. Although Mr. Thomas did not utilize topical corticosteroids for his treatment, there is a theoretical risk of excessive systemic absorption if patients apply and then occlude topical corticosteroids on a large body surface area for extended periods of time. To mitigate any medical-legal liability, providers can ask patients to sign an educational form confirming that the patient understands the risks and 
benefits of an at-home regimen, which has not been studied in a large cohort of patients. This is an approach used by physicians who prescribe home phototherapy. Additional studies are necessary to determine if home Goeckerman therapy is as effective and safe as its traditional counterpart.

Patient-centered treatment options are crucial for increasing access to medical treatment. The availability of at-home care for conditions such as psoriasis is an important goal. Home phototherapy units, for example, have proven to be successful despite initial concerns about safety and efficacy [11]. Additionally, preserving patient autonomy is fundamental to providing medical care within the context of a shared decision-making model. As such, physicians must not shy away from beneficial patient-directed treatments, particularly in cases in which patients are willing to adhere to at-home treatment and when at-home treatment can facilitate therapies that would otherwise be unavailable to patients or too burdensome to pursue. For patients to make informed decisions, all clinicians should provide the most upto-date and comprehensive information available regarding diagnoses and efficacy and safety profiles of all available therapeutic options. Patients who have interest and are motivated, such as Mr. Thomas, should also be included in the early stages of developing research proposals (i.e., clinical trials), systematic reviews, and clinical practice guidelines. Finally, as in Mr. Thomas' case, patient-directed treatments can also promote dialogue and community development between patients who share similar experiences and may benefit significantly from peer support. This article is based on previously conducted studies and does not contain any studies with human participants or animals performed by any of the authors.

\section{ACKNOWLEDGEMENTS}

Funding. No funding or sponsorship was received for this study or publication of this article.
Authorship. All named authors meet the International Committee of Medical Journal Editors (ICMJE) criteria for authorship for this article, take responsibility for the integrity of the work as a whole, and have given their approval for this version to be published.

Disclosures. Wilson Liao has received research grant funding from Abbvie, Amgen, Janssen, Novartis, Regeneron, Sanofi, and TRex Bio. Wilson Liao is also a member of the Editorial Board of Dermatology and Therapy. Tina Bhutani is a PI for Janssen, Merck, Celgene, and Regeneron and an advisor for Abbvie and Eli Lilly. Vidhatha Reddy, Shawn Thomas, Quinn Thibodeaux and Bridget Myers have nothing to disclose.

Compliance with Ethics Guidelines. This article is based on previously conducted studies and does not contain any studies with human participants or animals performed by any of the authors.

Data Availability. Data sharing is not applicable to this article as no datasets were generated or analyzed during the current study.

Open Access. This article is licensed under a Creative Commons Attribution-NonCommercial 4.0 International License, which permits any non-commercial use, sharing, adaptation, distribution and reproduction in any medium or format, as long as you give appropriate credit to the original author(s) and the source, provide a link to the Creative Commons licence, and indicate if changes were made. The images or other third party material in this article are included in the article's Creative Commons licence, unless indicated otherwise in a credit line to the material. If material is not included in the article's Creative Commons licence and your intended use is not permitted by statutory regulation or exceeds the permitted use, you will need to obtain permission directly from the copyright holder. To view a copy of this licence, visit http:// creativecommons.org/licenses/by-nc/4.0/. 


\section{REFERENCES}

1. Goeckerman WH. Treatment of psoriasis: continued observations on the use of crude coal tar and ultraviolet light. Arch Dermatol Syphilol. 1931;24(3):446-50.

2. Kim HO, Kim JH, Chung BY, Choi MG, Park CW. Increased expression of the aryl hydrocarbon receptor in patients with chronic inflammatory skin diseases. Exp Dermatol. 2014;23(4):278-81.

3. Sekhon S, Jeon C, Nakamura M, et al. Review of the mechanism of action of coal tar in psoriasis. J Dermatol Treat. 2018;29(3):230-2.

4. Serrao R, Davis MDP. Goeckerman treatment for remission of psoriasis refractory to biologic therapy. J Am Acad Dermatol. 2009;60(2):348-9.

5. Fitzmaurice S, Bhutani T, Koo J. Goeckerman regimen for management of psoriasis refractory to biologic therapy: the University of California San Francisco experience. J Am Acad Dermatol. 2013;69(4):648-9.

6. Lee E, Koo J. Modern modified 'ultra' Goeckerman therapy: a PASI assessment of a very effective therapy for psoriasis resistant to both prebiologic and biologic therapies. J Dermatol Treat. 2005;16(2): 102-7. https://doi.org/10.1080/095466305100331 40 .

7. Menter A, Cram DL. The Goeckerman regimen in two psoriasis day care centers. J Am Acad Dermatol. 1983;9(1):59-655. https://doi.org/10.1016/S01909622(83)70107-6.

8. Dennis M, Bhutani T, Koo J, Liao W. Goeckerman therapy for the treatment of eczema: a practical guide and review of efficacy. J Dermatol Treat. 2013;24(1):2-6.

9. Sorenson E, Levin E, Koo J, Berger TG. Successful use of a modified Goeckerman regimen in the treatment of generalized prurigo nodularis. J Am Acad Dermatol. 2015;72(1):e40-42.

10. Kaidbey KH, Kligman AM. Clinical and histological study of coal tar phototoxicity in humans. Arch Dermatol. 1977;113(5):592-5.

11. Koek MBG, Buskens E, van Weelden $H$, Steegmans PHA, Bruijnzeel-Koomen CAFM, Sigurdsson V. Home versus outpatient ultraviolet $B$ phototherapy for mild to severe psoriasis: pragmatic multicentre randomised controlled non-inferiority trial (PLUTO study). BMJ. 2009;7(338):b1542. 